\title{
Relationship between muscle strength and fall episodes among the elderly: the Yilan study, Taiwan
}

\author{
Nan-Ping Yang ${ }^{1,2^{*}+} \mathbb{D}$, Nai-Wei Hsu ${ }^{1,3+}$, Ching-Heng Lin ${ }^{1,4 \dagger}$, Hsi-Chung Chen ${ }^{1,5}$, Hsuan-Ming Tsao ${ }^{1,3}$, Su-Shun Lo ${ }^{1,6}$ \\ and Pesus Chou ${ }^{1 *}$
}

\begin{abstract}
Background: Fall episodes are not unusual among community residents, especially the elderly, and lower muscle strength is an important issue to address in order to prevent falls.

Methods: A community health survey was conducted in a suburban area of Taiwan, and 1067 older adults were selected for enrollment in the present study. All the enrolled subjects had been visited at their homes; the subjects' strength of both hands and muscle mass of both legs were measured and well-established questionnaires were finished by certificated paramedic staffs.

Results: The incidence of fall episodes in the previous 1 year in the Yilan elderly population was $15.1 \%$, and the female predominance was significant. A significantly higher prevalence of cataracts was found in group who experienced a fall in the past year (64\% vs. $54.9 \%$ in the non-fall group). Mild or more severe dementia was much more prevalent in the group who experienced a recent fall (33.8\% vs. $25.7 \%$ in the non-fall group). The strength of both hands tested as the physical function was $17.6 \pm 8.0 \mathrm{~kg}$ in the recent fall group, significantly weaker than that in the non-fall group $(20.7 \pm 8.7 \mathrm{~kg})$. Multivariate regression analysis revealed a greater weekly exercise duration and greater strength of both hands reduced the occurrence of falls among the whole and the female population. The standardized effect sizes of hand grip strength between both groups, not trivial, were 0.29 and 0.37 for the total population and the female subpopulation respectively.
\end{abstract}

Conclusions: Less weekly exercise duration and weaker muscle strength were f ound to be independent risk factors of fall episode(s) in an elderly Taiwanese population, especially in the female sub-population. Muscle strength, measured by average of both hands grip strength, was the most significantly factor of one-year fall episode(s) accessed retrospectively.

Keywords: Hand grip strength, Falling, Elderly population

\section{Background}

Fall episodes were not unusual in community residents, especially the elderly. Moreover, approximately $30 \%$ of people over 65 years of age living in the community fall each year, according to a review based on the Cochrane database [1]. Injuries sustained in a fall were more likely to be serious than those due to other reasons in community-dwelling

\footnotetext{
* Correspondence: yang.nanping@gmail.com; pschou@ym.edu.tw

${ }^{\dagger}$ Equal contributors

${ }^{1}$ Community Medicine Research Center \& Institute of Public Health, National Yang-Ming University, No.155, Sec.2, Linong Street, Taipei 112, Taiwan, Republic of China

Full list of author information is available at the end of the article
}

older adults that were characterized as hip fracture, head injury and/or other fracture [2]. From viewpoints of prevention medicine, all trials to reduce rate of falling or consequence of falling would be worthy. For an example, environmental improvements such as impact-absorbing flooring could significantly reduce the relative risk (RR) of injury in the event of a fall by $59 \%$ among the frail elderly in a nursing home [3]. Moreover, many studies had aimed to develop various exercise programs to reduce the risk of falling in older adults $[1,4]$. Therefore, exercise was expected to be of benefit to prevent falls and even subsequent injuries.

(C) The Author(s). 2018 Open Access This article is distributed under the terms of the Creative Commons Attribution 4.0 International License (http://creativecommons.org/licenses/by/4.0/), which permits unrestricted use, distribution, and 
.Poor lower extremity physical function, especially a poor walking ability, has been found to be an important risk factor related to falls in elderly community-dwelling people [5]. Usually, walking ability can be measured quantitatively by some timed walking tests such as Timed-Up \& Go [TUG], 10-m walk test [10MWT], 6-min walk test [6]. The predictive value of walking speed as an individual physical frailty indicator on activities of daily living (ADL) disability had been approved in community-dwelling elderly people [7] and, some elderly persons having low physical function and low self-confidence in terms of balance tend to overestimate their balance and ambulation function that might have a high risk of accidents [8]. However, in terms of a large-scale community survey, it would be necessary to find some more feasible measures rather than the above-mentioned functional tests that could be performed quickly and safely by the older population. There were two objectives could be considered.

First, low muscle mass (i.e. sarcopenia) could be defined as age-related lean tissue mass loss that usually resulted in reduced physical function, muscular strength, and mobility [9]. Reported prevalence of sarcopenia ranged widely depending on the reference cut-off thresholds for muscle mass and calf circumference; racial and geographic differences should be also taken into considerations [10]. Recently, sarcopenia could be defined using the Asian Working Group consensus algorithm, combining muscle mass, gait speed, and even grip strength and, adjusted by age and gender [11]. Second, low muscle strength was associated with poor physical function in older people, both male and female [12]. Hand grip strength was shown to be correlated with sarcopenia, physical function and walking ability [13, 14]. Additionally, grip strength was also a simple and inexpensive test, positively correlated with measures of nutritional health [15] and could be considered in clinical settings for identifying future falls and even fractures $[15,16]$.

It was rarely studied to investigate the influences of exercise, muscle mass, and muscle strength on falling, especially in an Asia elderly population. As a result, this study was a community-based survey to study the relationships between the above mentioned indicators (exercise, muscle mass, muscle strength) and fall episode(s) in an elderly Taiwanese population.

\section{Methods}

\section{Study design and participants}

The study was part of the Yilan study [17, 18], a population-based community health survey conducted by the Community Medicine Research Center at the National Yang-Ming University and the National Yang-Ming University Hospital in Taiwan. The protocol was evaluated and approved by the Institutional Review Board (IRB) of National Yang-Ming University Hospital (IRB Approval No.: 2011A016), which has been certificated by the Ministry of
Health and Welfare, Taiwan. All the enrolled participants provided written informed consent.

From August 2013 to February 2016, all residents aged 65 years or more living in Yilan City, located in northeast Taiwan, were randomly selected to participate. All participated subjects had been visited at their homes, and wellestablished questionnaires [19], approved by the IRB of National Yang-Ming University Hospital, were used to collect their basic data and health-related information including demographic characteristics, body weight and height, education level, living status, lifestyle, smoking and drinking habits, self-reported medical histories (i.e., history of various chronic diseases), exercise condition within past 1 week, sleep status and quality of life within past 1 month, falling episode within past 1 year and current psychological status (i.e., dementia measured by the ascertain dementia 8item informant questionnaire (AD8) [20]) that were administered by trained project assistants through face to face interviews. Excluding the individuals who failed to provide a past medical history, who could not complete interview, or who were unable to cooperate with the collection of anthropometric data or physical functional test, finally there were 1067 older adults enrolled in the present study.

\section{Measurements of hand grip strength and muscle mass}

The strength of both hands and the muscle mass of both legs were also measured by certificated paramedic staff of the National Yang-Ming University Hospital in Yilan, Taiwan, using a hydraulic hand dynamometer (Jamar, Jackson, MI, USA) and an InnerScan segmental body composition analyzer (InnerScan V,TANITA ${ }^{\bullet}$ BC601, Japan) with an operating frequency of $50 \mathrm{kHz}$ at $100 \mu \mathrm{A}$ respectively. Every participant completed two trials for each hand and, the best performance of each hand was averaged as the final estimate of hand grip strength [19]. The estimation of segmental muscle mass for mode BC601 has been validated [21]; the estimated lean mass $(\mathrm{kg})$ for each lower extremity was averaged as the final estimate.

\section{Statistical analysis}

Descriptive statistics were presented as numbers of cases, percentages, and means with standard deviation (SD). The independent t-test and the chi-square test were used to analyze significant differences between groups (group I: population without any fall episode; group II: population with fall episode(s)) for continuous and categorical variables. Adjusted odds ratios (AORs) and $95 \%$ confidence intervals (CIs) were calculated by the logistic regression method to perform multivariate analyses. Furthermore, the magnitude of effects (i.e. effect size (ES) statistics) of those significantly effective independent variables was also calculated to assess the 
relationships within data [22]. The formula of effect size is as below [23]:

Standardized ES $=([$ mean of group 1$]-[$ mean of group 2] $)$

$$
/ \mathrm{SD}_{\text {(pooled) }}
$$

In addition, the Cohen's $f^{2}$ effect size measure for multiple regression is defined as:

$$
f^{2}=R^{2} / 1-R^{2},
$$

where $R^{2}$ is the squared multiple correlation [24].

All analyses of data were conducted using the Statistical Package for Social Sciences for Windows (SPSS Ver.22.0). All reported $p$ values are two-tailed, and were considered significant if $p \leq 0.05$.

\section{Results}

The basic characteristics of all the enrolled subjects are presented in Table 1. The study included 1067 people aged 65 years or more (mean age: $76.4 \pm 6.0$ years), 439 males (41.1\%) and 628 females (58.9\%). Only a small proportion of the studied subjects lived alone, $4.4 \%$ for part of the time and $6.8 \%$ full-time. The prevalence of

Table 1 Basic characteristics of the enrolled subjects and their

\begin{tabular}{|c|c|c|}
\hline & \multicolumn{2}{|c|}{ Total $(N=1067)$} \\
\hline & No., Mean & $\%$, SD \\
\hline \multicolumn{3}{|l|}{ Gender (male) } \\
\hline male & 439 & 41.1 \\
\hline female & 628 & 58.9 \\
\hline Age (years) & 76.4 & 6.0 \\
\hline \multicolumn{3}{|l|}{ Living Status } \\
\hline full-time living alone & 73 & 6.8 \\
\hline part-rime living alone & 47 & 4.4 \\
\hline not living alone & 947 & 88.8 \\
\hline \multicolumn{3}{|l|}{ Chronic Physical Disorders } \\
\hline DM (yes) & 263 & 24.6 \\
\hline HT (yes) & 626 & 58.7 \\
\hline CAD (yes) & 366 & 34.3 \\
\hline CVA (yes) & 29 & 2.7 \\
\hline Cataract (yes) & 600 & 56.2 \\
\hline \multicolumn{3}{|l|}{ Cognitive Impairment (by CDR score) } \\
\hline mild or more severe dementia $(\geqq 2)$ & 228 & 27.0 \\
\hline Fall episode within the past year (yes) & 161 & 15.1 \\
\hline Strength $(\mathrm{kg})$, average of both hands & 20.2 & 8.7 \\
\hline Muscle mass $(\mathrm{kg})$, average of both legs & 7.3 & 1.8 \\
\hline Exercise (mins), accumulated in 1 week & 182 & 223 \\
\hline
\end{tabular}
experiences of falls within the past 1 year

$D M$ diabetes mellitus, $H T$ hypertension, CAD coronary arterial disease, CVA cerebrovascular disease various chronic physical disorders related to accidental falls, including diabetes mellitus (24.6\%), hypertension $(58.7 \%)$, coronary arterial disease $(34.3 \%)$, cerebrovascular disease $(2.7 \%)$ and cataracts $(56.2 \%)$, were also investigated. Using the clinical dementia rating (CDR) method, evaluated based on AD8, to identify the cognitive impairment status of the elderly subjects, 228 subjects $(27.0 \%)$ were considered to have mild or more severe dementia. Within 1 year, the incidence of falls, which was accessed retrospectively, in the studied Yilan elderly population, was $15.1 \%$, and their exercise duration was $182 \pm 223 \mathrm{~min}$ per week. When visited at home, the strength of both hands and the muscle mass of both legs of the enrolled subjects were measured to be $20.2 \pm 8.7$ and $7.3 \pm 1.8 \mathrm{~kg}$, respectively.

The study population was divided into two subgroups, a fall group (those who had experienced a fall within the past year) and a non-fall group (those who had not experienced a fall in the past year), and the characteristics of the two different populations were compared (as shown in Table 2). A significantly greater proportion of female subjects experienced a fall episode within the past year, accounting for $68.9 \%$ of the fall subgroup (as compared with $57.1 \%$ of the non-fall group; $p=0.005$ ). A significantly higher prevalence of cataracts was also found in the fall group (64\%, as compared with $54.9 \%$ in the nonfall group; $p=0.03$ ). Mild or more severe dementia, as indicated by a CDR score $\geq 2$, was much more prevalent in the fall group than the non-fall group $(33.8 \%$ vs. 25 . $7 \% ; p=0.05)$. With regards to the physical function tests, only the strength of both hands differed significantly between groups, at $17.6 \pm 8.0 \mathrm{~kg}$ in the fall group vs. $20.7 \pm 8.7 \mathrm{~kg}$ in the non-fall group $(p<0.0001)$.

A multivariate logistic regression model was used to analyze the factors associated with a fall episode within the past year among the studied elderly population of Yilan (Table 3). A greater duration of exercise per week was shown to reduce the occurrence of fall(s) significantly among the whole study population (AOR $0.999,95 \%$ CI 0. 998-1.000; standardized ES 0.15), and particularly in the female subgroup (AOR 0.999, 95\% CI 0.997-1.000; standardized ES 0.23). A greater strength of average of both hands was also shown to reduce the occurrence of fall(s) significantly among the whole study population (AOR 0.95 , 95\% CI 0.92-0.98; standardized ES 0.37), and particularly in the female subgroup (AOR 0.94, 95\% CI 0.90-0.99; standardized ES 0.29). A small effect of 0.2 is noticeably smaller than medium $(\mathrm{ES}=0.5)$ but not so small as to be trivial [23]. That meant that the elder community-dwelling Taiwanese adults without any fall episode within the past 1 year had a higher score of hand grip strength than 58-69\% of the others [23]. Furthermore, the Cohen's $f^{2}$ effect size measures for multiple regressions were calculated that effect size for full model in female population was 0.057 
Table 2 Bivariate analysis of cases with fall episode(s) within the past 1 year compared to the others

\begin{tabular}{|c|c|c|c|c|c|}
\hline & \multicolumn{2}{|c|}{ Population without any fall episode } & \multicolumn{2}{|c|}{ Population with fall episode(s) } & \multirow[t]{2}{*}{$P$ value of $x^{2}$ test or $t$ test } \\
\hline & No., Mean & $\%$, SD & No., Mean & $\%, \mathrm{SD}$ & \\
\hline \multicolumn{6}{|l|}{ Gender } \\
\hline male & 389 & 42.9 & 50 & 31.1 & \multirow[t]{2}{*}{0.005} \\
\hline female & 517 & 57.1 & 111 & 68.9 & \\
\hline Age (years) & 76.3 & 6.0 & 76.8 & 6.0 & 0.37 \\
\hline \multicolumn{6}{|l|}{ Living Status } \\
\hline full-time living alone & 56 & 6.2 & 17 & 10.6 & \multirow[t]{3}{*}{0.08} \\
\hline part-rime living alone & 38 & 4.2 & 9 & 5.6 & \\
\hline not living alone & 812 & 89.6 & 135 & 83.9 & \\
\hline \multicolumn{6}{|l|}{ Chronic Physical Disorders } \\
\hline DM (yes) & 222 & 24.5 & 41 & 25.5 & 0.79 \\
\hline HT (yes) & 530 & 58.5 & 96 & 59.6 & 0.79 \\
\hline CAD (yes) & 311 & 34.3 & 55 & 34.2 & 0.97 \\
\hline CVA (yes) & 25 & 2.8 & 4 & 2.5 & 0.84 \\
\hline Cataract (yes) & 497 & 54.9 & 103 & 64.0 & 0.03 \\
\hline \multicolumn{6}{|l|}{ Cognitive Impairment (by CDR score) } \\
\hline mild or more severe dementia $(\geqq 2)$ & 183 & 25.7 & 45 & 33.8 & 0.05 \\
\hline Strength $(\mathrm{kg})$, average of both hands & 20.7 & 8.7 & 17.6 & 8.0 & $<0.0001$ \\
\hline Muscle mass $(\mathrm{kg})$, average of both legs & 7.3 & 1.8 & 7.1 & 2.1 & 0.14 \\
\hline Exercise (mins), accumulated in 1 week & 187 & 226 & 153 & 201 & 0.08 \\
\hline
\end{tabular}

CDR clinical dementia rating, $\mathrm{kg}$ kilograms, mins minutes

Table 3 Multivariate analysis of fall episode(s) within the past 1 year

\begin{tabular}{|c|c|c|c|c|c|c|c|c|}
\hline & \multicolumn{2}{|c|}{ Male Population } & \multicolumn{3}{|c|}{ Female Population } & \multicolumn{3}{|c|}{ Total Population } \\
\hline & AOR & $95 \% \mathrm{Cl}$ & AOR & $95 \% \mathrm{Cl}$ & St. $E^{a}$ & AOR & $95 \% \mathrm{Cl}$ & St. $E S^{a}$ \\
\hline Gender (male) & - & & - & & & 0.80 & $0.40-1.58$ & \\
\hline Age (years) & 1.02 & $0.95-1.08$ & 0.96 & $0.92-1.01$ & & 0.99 & $0.95-1.02$ & \\
\hline \multicolumn{9}{|l|}{ Living Status } \\
\hline full-time living alone & Ref. & & Ref. & & & Ref. & & \\
\hline part-rime living alone & 0.36 & $0.03-4.18$ & 1.99 & $0.60-6.57$ & & 1.19 & $0.43-3.32$ & \\
\hline not living alone & 0.40 & $0.11-1.38$ & 0.75 & $0.33-1.68$ & & 0.68 & $0.35-1.32$ & \\
\hline \multicolumn{9}{|l|}{ Chronic Physical Disorders } \\
\hline DM (yes) & 1.78 & $0.84-3.76$ & 0.73 & $0.41-1.32$ & & 0.97 & $0.62-1.51$ & \\
\hline HT (yes) & 0.91 & $0.43-1.89$ & 0.89 & $0.54-1.47$ & & 0.90 & $0.60-1.36$ & \\
\hline CAD (yes) & 1.08 & $0.52-2.26$ & 0.58 & $0.33-1.00$ & & 0.68 & $0.44-1.05$ & \\
\hline CVA (yes) & 2.38 & $0.55-10.3$ & 0.26 & $0.03-2.17$ & & 0.85 & $0.28-2.58$ & \\
\hline Cataract (yes) & 1.08 & $0.54-2.15$ & 1.55 & $0.93-2.57$ & & 1.35 & $0.90-2.02$ & \\
\hline \multicolumn{9}{|l|}{ Cognitive Impairment (by CDR score) } \\
\hline mild or more severe dementia $(\geqq 2)$ & 1.01 & $0.47-2.17$ & 1.51 & $0.89-2.55$ & & 1.29 & $0.85-1.95$ & \\
\hline Strength $(\mathrm{kg})$, average of both hands & 0.96 & $0.92-1.01$ & 0.94 & $0.90-0.99$ & 0.29 & 0.95 & $0.92-0.98$ & 0.37 \\
\hline Muscle mass $(\mathrm{kg})$, average of both legs & 1.12 & $0.92-1.38$ & 1.01 & $0.75-1.37$ & & 1.12 & $0.95-1.32$ & \\
\hline Exercise (mins), accumulated in 1 week & 1.001 & $0.999-1.002$ & 0.999 & $0.997-1.000$ & 0.23 & 0.999 & $0.998-1.000$ & 0.15 \\
\hline
\end{tabular}


and in total population was 0.038 . By convention, $f^{2}$ effect sizes of $0.02,0.15$, and 0.35 are termed small, medium, and large, respectively [24]. Similarly, the effect size from the regression models is still noticeably smaller than medium but not so small as to be trivial. Otherwise, among the medical histories, only positive CAD status was an associated factor of falling in the female subpopulation (AOR 0.58, 95\% CI 0.33-1.00).

Summarily, muscle strength, measured by average of both hands grip strength, was the most significantly independent factor of one-year fall episode(s) accessed retrospectively among the elderly in a Taiwan's community.

\section{Discussion}

In Taiwan, an urban community surveillance of residents aged 65 years or over in the capital city showed that $16.3 \%$ of the elderly persons interviewed had experienced at least one fall in the past 1 year, and gender, visual impairment and orthostatic hypotension were identified as major risk factors of fall injuries in the elderly [25]. A total of $53.4 \%$ of the participants reported a fear of falling [26], and fear of falling was identified as a major factor influencing healthrelated quality of life in the same elderly population [27]. In the present study, performed in a suburban community in Taiwan, the incidence of falling in the past year was 15. $1 \%$ with its $95 \%$ CI $(13.0-17.2 \%)$ that was equivalent as compared with the abovementioned previous study.

After systematic reviewing of literatures, group and home-based exercise programs, and home safety interventions were well-known to reduce rate of falls and risk of falling [1]. Furthermore, exercise interventions in community-dwelling older people also could probably reduce fear of falling to a limited extent immediately after the intervention, without increasing the risk or frequency of falls [28]. In the present study, the exercise behavior (i.e. duration of exercise per week) was also found to be a potentially protective factor of falling episode(s) for the community-dwelling older adults in Taiwan although it was not statistically significant $(p=0.08)$ in the stage of bivariate analysis. However, it was still an important factor of falling in the elderly after adjusting other variates in the final multivariate logistic model.

In the present study, another meaningful finding was that mild or more severe dementia, as indicated by a CDR score $\geq 2$, was much more prevalent in the fall group than the non-fall group $(33.8 \%$ vs. $25.7 \%$; $p=0.05)$. In another study in Taiwan, 280 Taiwanese people aged 65-91 years who were not taking antidepressant medication were followed-up for 2 years with monthly telephone calls to determine the incidence of falls: $19.6 \%$ of the participants had experienced a fall on one occasion, and $13.5 \%$ had fallen two or more times. Depressive symptoms were significantly more prevalent in the recurrent fallers (40.0\%) and the once-only fallers (27.5\%) than in the nonfallers (16.1\%) [29]. Findings of the two studies in similar geographic areas were still similar.

Furthermore, in the present study, a medical history of cataract was still noted to be a risk factor of falling episode(s) for the community-dwelling older adults in Taiwan in the stage of bivariate analysis. Poor vision was known to reduce postural stability and significantly increase the risk of falls and fractures in older people [30]. A systematic review of the literature concluded that expedited cataract surgery was effective in significantly enhancing vision (OR, 7.22; 95\% CI, 3.16-16.55; $P<.0001)$ but was inconclusive in preventing falls (OR, $0.81 ; 95 \% \mathrm{CI}, 0.55-1.17)$ [31]. It would be worthy to further study the relationship between visual problems and falling in the elderly.

Sarcopenia had been shown to be associated with an increase in the risk of falling or physical disability in community-dwelling middle-aged and older adults in several studies [32-37]. However, in the present study, a cross-sectional survey of suburban community-dwelling elderly people in Yilan, Taiwan, showed that there was no difference in the muscle mass of both legs between the elderly subjects who had and had not experienced a fall in the past year. It would be possible due to the healthy effect because the randomly selected subjects who were unable to cooperate with the collection of anthropometric data or physical functional test were excluded from the present study. Further long-term follow-up study of the subjects enrolled or excluded in the present study and comparisons among urban-, suburban- and rural-community living elderly people are necessary to be assessed in depth.

On the other hand, appendicular skeletal muscle mass was found to be significantly correlated with handgrip strength in elderly Chinese subjects [38]; additionally, hand grip strength had been reviewed and concluded to be used for the diagnosis of sarcopenia and frailty [39]. Focused on older people, many recent studies had concluded that strength measures, including peak torque of flexors and extensors of the knee and hip joints, maximum voluntary isometric strength (MVIS) of hip abductors, and hand grip strength, were better than muscle mass measures in terms of predicting health-related outcomes such as balance, functional mobility, daily physical activities and falls [40-43]; both hand grip strength and knee extension strength, highly correlated and internally consistent, were commonly used to measure muscle power $[44,45]$. Many measured physical tests, including grip strength (GS), the chair stand test (CST), one-leg standing test (OLS), TUG, 10MWT, 6-m walking speed at a comfortable pace (CWS) and at maximum pace (MWS), and TUG, even self-reported walking ability, had been used to screen fall risk and functional activities in frail older people [6, 46-48]. Summarily, measurements of both 
hand grip forces by the dynamometry were more convenient and well established as an indicator of muscle status, particularly among older adults. In the present study, the strength of both hands was significantly weaker in the fall group $(17.6 \pm 8.0 \mathrm{~kg})$ than the nonfall group $(20.7 \pm 8.7 \mathrm{~kg}, p<0.0001)$. At the final multivariate logistic model, hand strength was the most significantly independent factor of one-year fall episode(s). This finding should encourage health promotion activities for the elderly in communities in Taiwan and other countries. Therefore, in terms of a large-scale community survey such as the present study, hand grip strength would be the most feasible measure in the older population rather than other functional test.

\section{Conclusion}

The prevalence of fall episodes in the past 1 year in the Yilan elderly population was $15.1 \%$, and a shorter weekly exercise duration and weaker strength of both hands were independent risk factors related to falls in the total population and in the female sub-population. Especially, muscle strength, measured by average of both hands grip strength, was the most significantly independent factor of one-year fall episode(s) accessed retrospectively among the elderly in a Taiwan's community.

\section{Abbreviations \\ 10MWT: 10-m walk test; AD8: Ascertain dementia 8-item informant questionnaire; ADL: Activities of daily living; AOR: Adjusted odds ratios; CDR: Clinical dementia rating; Cls: Confidence intervals; CST: Chair stand test; CWS: Walking speed at a comfortable pace; ES: Effect size; EWGSOP: European working group on sarcopenia in older people; GS: Grip strength; IRB: Institutional review board; MVIS: Maximum voluntary isometric strength; MWS: Walking speed at maximum pace; OLS: One-leg standing test; RR: Relative risk; SD: Standard deviation; TUG: Timed up-and-go test}

\section{Acknowledgements}

The authors would like to thank the Community Angels (social volunteers) and the Yang-Ming Crusade (student volunteers of the National Yang-Ming University). The article-processing-charge was supported by grants from Taichung Veterans Hospital (TCVGH-YM1070203).

\section{Funding}

The present study was partially supported financially by National Yang-Ming University Hospital. The National Yang-Ming University Hospital had no role in the study design, data collection, analysis, interpretation and writing the manuscript.

\section{Availability of data and materials}

The datasets used and/or analyzed during the current study are available from the two co-corresponding authors on reasonable request.

\section{Authors' contributions}

The study was designed by PC, SSL and NWH; data were gathered and analyzed by NWH, HMT, SSL and CHL; the initial draft of the manuscript was written by NPY, NWH and CHL; the accuracy of the data and analyses was assured by HCC, HMT, SSL and PC. All authors participated in the preparation of the manuscript and approved the final version. All authors have read and approved the final manuscript. In general, NPY, NWH and CHL contributed equally to the work.

\section{Ethics approval and consent to participate}

The Institutional Review Board (IRB) of National Yang-Ming University Hospital, Taiwan, approved this study. All the enrolled participants provided written informed consent.

\section{Competing interests}

The authors declare that they have no competing interests.

\section{Publisher's Note}

Springer Nature remains neutral with regard to jurisdictional claims in published maps and institutional affiliations.

\section{Author details \\ ${ }^{1}$ Community Medicine Research Center \& Institute of Public Health, National Yang-Ming University, No.155, Sec.2, Linong Street, Taipei 112, Taiwan, Republic of China. ${ }^{2}$ Department of Orthopedics \& Surgery, Keelung Hospital, Ministry of Health \& Welfare, Keelung, Taiwan. ${ }^{3}$ Department of Intern Medicines, National Yang-Ming University Hospital, Yilan, Taiwan. ${ }^{4}$ Department of Medical Research, Taichung Veterans General Hospital, Taichung, Taiwan. ${ }^{5}$ Department of Psychiatry \& Center of Sleep Disorders, National Taiwan University Hospital, Taipei, Taiwan. ${ }^{6}$ Department of Surgery, National Yang-Ming University Hospital, Yilan, Taiwan.}

Received: 17 September 2017 Accepted: 3 April 2018

Published online: 13 April 2018

\section{References}

1. Gillespie LD, Robertson MC, Gillespie WJ, Sherrington C, Gates S, Clemson LM, Lamb SE. Interventions for preventing fall in older people living in the community. Cochrane Database Syst Rev. 2012;9:CD007146.

2. Xu D, Drew JA. Cause, nature and care-seeking behavior for injuries among community-dwelling older adults, USA, 2004-2013. Inj Prev. 2016;22:46-51.

3. Gustavsson J, Bonander C, Andersson R, Nilson F. Investigating the fall-injury reducing effect of impact absorbing flooring among female nursing home residents: initial results. Inj Prev. 2015;21:320-4.

4. El-Khoury F, Cassou B, Charles MA, Dargent-Molina P. The effect of fall prevention exercise programmes on fall induced injuries in community dwelling older adults: systematic review and meta-analysis of randomized controlled trials. BMJ. 2013;347:f6234.

5. Morita M, Takamura N, Kusano Y, Abe Y, Moji K, Takemoto T, Aoyagi K. Relationship between falls and physical performance measures among community-dwelling elderly women in Japan. Aging Clin Exp Res. 2005;17:211-6.

6. van Hedel HJ, Wirz M, Dietz V. Assessing walking ability in subjects with spinal cord injury: validity and reliability of 3 walking tests. Arch Phys Med Rehabil. 2005;86:190-6.

7. Vermeulen J, Neyens JC, van Rossum E, Spreeuwenberg MD, de Witte LP. Predicting ADL disability in community-dwelling elderly people using physical frailty indicators: a systematic review. BMC Geriat. 2011;11:33.

8. Sakamoto Y, Ohashi Y. The relationship between physical function in the elderly and judgment error in walking speed. J Phys Ther Sci. 2017;29:1176-80.

9. Gray M, Glenn JM, Binns A. Predicting sarcopenia from functional measures among community-dwelling older adults. Age. 2016;38:22.

10. Bahat G, Tufan A, Tufan F, Kilic C, Akpinar TS, Kose M, Erten N, Karan MA, Cruz-Jentoft AJ. Cut-off points to identify sarcopenia according to European Working Group on Sarcopenia in Older People (EWGSOP) definition. Clin Nutr. 2016;35:1557-63.

11. Jang IY, Jung HW, Lee CK, Yu SS, Lee YS, Lee E. Comparisons of predictive values of sarcopenia with different muscle mass indices in Korean rural older adults: a longitudinal analysis of the aging study of PyeongChang rural area. Clin Interv Aging. 2018;13:91-9.

12. Visser M, Deeg DJ, Lips P, Harris TB, Bouter LM. Skeletal muscle mass and muscle strength in relation to lower-extremity performance in older men and women. J Am Geriatr Soc. 2000;48:381-6.

13. Lino VT, Rodrigues NC, O'Dwyer G, Andrade MK, Mattos IE, Portela MC. Handgrip strength and factors associated in poor elderly assisted at a primary care unit in Rio de Janeiro, Brazil. PLoS One. 2016;11:e0166373.

14. Beseler MR, Rubio C, Duarte E, Hervás D, Guevara MC, Giner-Pascual M, Viosca E. Clinical effectiveness of grip strength in predicting ambulation of elderly inpatients. Clin Interv Aging. 2014;9:1873-7. 
15. Miller MD, Giles LC, Crotty M, Harrison JE, Andrews GR. A clinically relevant criterion for grip strength: relationship with falling in a sample of older adults. Nutr Diet. 2003;60:248-52.

16. Balogun S, Winzenberg T, Wills K, Scott D, Jones G, Aitken D, Callisaya ML. Prospective associations of low muscle mass and function with 10-year falls risk, incident fracture and mortality in community-dwelling older adults. J Nutr Health Aging. 2017;21:843-8.

17. Hsu NW, Tsao HM, Chen HC, Chou P. Anxiety and depression mediate the health-related quality of life differently in patients with cardiovascular disease and stroke-preliminary report of the Yilan study: a population-based community health survey. PLoS One. 2014;9:e107609.

18. Hsu NW, Tsao HM, Chen HC, Lo SS, Chen SA, Chou P. Different impacts of atrial fibrillation and cardiac premature contractions on the health-related quality of life in elderly people: the Yilan study. Tohoku J Exp Med. 2016;238:75-83.

19. Chen HC, Hsu NW, Chou P. The association between sleep duration and hand grip strength in community-dwelling older adults: the Yilan study, Taiwan. Sleep. 2017; https://doi.org/10.1093/sleep/zsx021.

20. Chen SF, Liu MH, Chen NC, Horng HD, Tsao WL, Chang CC, Chang YT. Educational effects on ascertain dementia 8-item informant questionnaire to detect dementia in the Taiwanese population. Int Psychogeriatr. 2017;10:1-9.

21. Pietrobelli A, Rubiano F, St-Onge MP, Heymsfield SB. New bioimpedance analysis system: improved phenotyping with whole-body analysis. Eur J Clin Nutr. 2004;58:1479-84

22. Hopkins WG, Marshall SW, Batterham AM, Hanin J. Progressive statistics for studies in sports medicine and exercise science. Med Sci Sports Exerc. 2009:41:3-13.

23. Sullivan GM, Feinn R. Using effect size-or why the $P$ value is not enough J Grad Med Educ. 2012:4:279-82.

24. Cohen J. Statistical power analysis for the behavioral sciences. 2nd ed. Hillsdale: Lawrence Erlbaum Associates, Publishers; 1988.

25. Chang NT, Yang NP, Chou P. Incidence, risk factors and consequences of falling injuries among the community-dwelling elderly in Shihpai, Taiwan. Aging Clin Exp Res. 2010;22:70-7.

26. Chang HT, Chen HC, Chou P. Factors associated with fear of falling among community-dwelling older adults in the Shih-Pai study in Taiwan. PLoS One. 2016;11:e0150612.

27. Chang NT, Chi LY, Yang NP, Chou P. The impact of falls and fear of falling on health-related quality of life in Taiwanese elderly. J Community Health Nurs. 2010:27:84-95.

28. Kendrick D, Kumar A, Carpenter H, Zijlstra GA, Skelton DA, Cook JR, Stevens Z, Belcher CM, Haworth D, Gawler SJ, Gage H, Masud T, Bowling A, Pearl M, Morris RW, lliffe S, Delbaere K. Exercise for reducing fear of falling in older people living in the community. Cochrane Database Syst Rev. 2014;11: CD009848.

29. Kwan MM, Lin SI, Close JC, Lord SR. Depressive symptoms in addition to visual impairment, reduced strength and poor balance predict falls in older Taiwanese people. Age Ageing. 2012;41:606-12.

30. Lord SR. Visual risk factors for falls in older people. Age Ageing. 2006; 35(Suppl 2):ii42-5.

31. Desapriya E, Subzwari S, Scime-Beltrano G, Samayawardhena LA, Pike I. Vision improvement and reduction in falls after expedited cataract surgery: systematic review and meta-analysis. J Cataract Refract Surg. 2010;36:13-9.

32. Landi F, Liperoti R, Russo A, Giovannini S, Tosato M, Capoluongo E, Bernabei $R$, Onder $G$. Sarcopenia as a risk factor for falls in elderly individuals: results from the ilSIRENTE study. Clin Nutr. 2012;31:652-8.

33. Beaudart C, Reginster JY, Petermans J, Gillain S, Quabron A, Locquet M, Slomian J, Buckinx F, Bruyère O. Quality of life and physical components linked to sarcopenia: the SarcoPhAge study. Exp Gerontol. 2015;69:103-10.

34. Tanimoto $Y$, Watanabe M, Sun W, Sugiura Y, Hayashida I, Kusabiraki T, Tamaki J. Sarcopenia and falls in community-dwelling elderly subjects in Japan: defining sarcopenia according to criteria of the European working group on sarcopenia in older people. Arch Gerontol Geriatr. 2014;59:295-9.

35. Scott D, Hayes A, Sanders KM, Aitken D, Ebeling PR, Jones G. Operational definitions of sarcopenia and their associations with 5-year changes in falls risk in community-dwelling middle-aged and older adults. Osteoporos Int. 2014;25:187-93.

36. Bischoff-Ferrari HA, Orav JE, Kanis JA, Rizzoli R, Schlögl M, Staehelin HB, Willett WC, Dawson-Hughes B. Comparative performance of current definitions of sarcopenia against the prospective incidence of falls among community-dwelling seniors age 65 and older. Osteoporos Int. 2015;26:2793-802
37. Tanimoto Y, Watanabe M, Sun W, Tanimoto K, Shishikura K, Sugiura Y Kusabiraki T, Kono K. Association of sarcopenia with functional decline in community-dwelling elderly subjects in Japan. Geriatr Gerontol Int. 2013;13:958-63.

38. Zeng P, Han Y, Pang J, Wu S, Gong H, Zhu J, Li J, Zhang T. Sarcopenia-related features and factors associated with lower muscle strength and physical performance in older Chinese: a cross sectional study. BMC Geriatr. 2016;16:45.

39. Sousa-Santos AR, Amaral TF. Differences in handgrip strength protocols to identify sarcopenia and frailty - a systematic review. BMC Geriatr. 2017;17:238

40. Samuel D, Rowe P. An investigation of the association between grip strength and hip and knee joint moments in older adults. Arch Gerontol Geriatr. 2012;54:357-60.

41. Gafner SC, Bastiaenen CH, Ferrari S, Gold G, Terrier P, Hilfiker R, Allet L. Hip muscle and hand-grip strength to differentiate between older fallers and non-fallers: a cross-sectional validity study. Clin Interv Aging. 2017;13:1-8.

42. Menant JC, Weber F, Lo J, Sturnieks DL, Close JC, Sachdev PS, Brodaty H, Lord SR. Strength measures are better than muscle mass measures in predicting health-related outcomes in older people: time to abandon the term sarcopenia? Osteoporos Int. 2017:28:59-70.

43. Hwang AC, Zhan YR, Lee WJ, Peng LN, Chen LY, Lin MH, Liu LK, Chen LK. Higher daily physical activities continue to preserve muscle strength after midlife, but not muscle mass after age of 75. Medicine (Baltimore). 2016;95:e3809.

44. Bohannon RW, Magasi SR, Bubela DJ, Wang YC, Gershon RC. Grip and knee extension muscle strength reflect a common construct among adults. Muscle Nerve. 2012:46:555-8.

45. Bohannon RW. Muscle strength: clinical and prognostic value of hand-grip dynamometry. Curr Opin Clin Nutr Metab Care. 2015;18:465-70.

46. Alexander NB, Guire KE, Thelen DG, Ashton-Miller JA, Schultz AB, Grunawalt JC, Giordani B. Self-reported walking ability predicts functional mobility performance in frail older adults. J Am Geriatr Soc. 2000:48:1408-13.

47. Julius LM, Brach JS, Wert DM, VanSwearingen JM. Perceived effort of walking: relationship with gait, physical function and activity, fear of falling, and confidence in walking in older adults with mobility limitations. Phys Ther. 2012:92:1268-77.

48. Shimada H, Suzukawa M, Tiedemann A, Kobayashi K, Yoshida H, Suzuki T. Which neuromuscular or cognitive test is the optimal screening tool to predict falls in frail community-dwelling older people? Gerontology. 2009;55:532-8.

\section{Ready to submit your research? Choose BMC and benefit from:}

- fast, convenient online submission

- thorough peer review by experienced researchers in your field

- rapid publication on acceptance

- support for research data, including large and complex data types

- gold Open Access which fosters wider collaboration and increased citations

- maximum visibility for your research: over $100 \mathrm{M}$ website views per year

At BMC, research is always in progress.

Learn more biomedcentral.com/submissions 